\title{
Rifampin Induced Acute Tubulo-interstitial Nephritis: Rare Event of Commonly Used Drug
}

\author{
Madhuri Rudrarajuㅁ, Varun Parvathagiri ${ }^{1}$, Manish Dugar ${ }^{2}$ \\ ${ }^{1}$ Pharm.D Interns, Sri Venkateshwara College of Pharmacy, Affiliated to Osmania University, Hyderabad $;{ }^{2}$ Consultant Rheumatologist, \\ Apollo Hospitals, Hyderabad, India.
}

\section{Corresponding Author:}

Dr. Madhuri Rudraraju

Email: madhu.rudraraju5@gmail.com

This is an Open Access article distributed under the terms of the Creative Commons Attribution License (creativecommons.org/ licenses/by/3.0).

Received : January 2, 2017

Accepted : March 7, 2017

Published : April 10, 2017

\begin{abstract}
Background: Interstitial nephritis, complication of anti-tubercular therapy has been most commonly reported due to rifampin therapy. The mechanism of rifampin-induced acute kidney injury is either a type II or type III hypersensitivity reaction induced by rifampin antigens in which anti-rifampin antibodies form immune complexes that are deposited in renal vessels, the glomerular endothelium, and the interstitial area. Case Report: We describe a case of 57-year-old man with rheumatoid arthritis who developed acute kidney injury with prominent tubular dysfunction after starting anti-tubercular therapy containing rifampin. Conclusion: Acute tubulo-interstitial nephritis should be included in the differential diagnosis in acute kidney injury and renal tubular damage following rifampin initiation.
\end{abstract}

Keywords: Acute Kidney Injury, Acute Tubulointerstitial Nephritis, Kidney Tubules, Nephritis, Rifampin.

\section{Introduction}

Rifampin is one of the key drugs that has helped to shorten the treatment duration in tuberculosis (TB). Serious adverse reactions due to rifampin are uncommon. Minor side effects like nausea and vomiting can be managed with symptomatic treatment. Rarely major adverse reactions endangering life such as thrombocytopenia [1] and acute renal failure [2] may develop. Acute renal failure due to rifampin therapy though uncommon has been described since 1971 [3]. It is usually reversible if detected early and treated appropriately. ATIN (Acute Tubular Interstitial Nephritis) as a complication of anti-tubercular therapy has been most commonly reported due to rifampin therapy. This reaction typically occurs following re-exposure to the drug.

The Tuberculosis Research Centre (TRC) of the Indian Council of Medical Research in more than 30 controlled clinical trials have treated more than 8000 patients with pulmonary or extrapulmonary tuberculosis with rifampin containing regimens with reporting of only three cases of rifampin induced acute renal failure [4,5]. In the current case, we are going to report rifampin induced acute tubulo-interstitial nephritis, which is a rare adverse event of most commonly used drug.

\section{Case Report}

A 57 year old male weighing $65 \mathrm{~kg}$ with a past history of rheumatoid arthritis (RA), hypertension, asthma and pulmonary tuberculosis presented with pain and synovitis in the left ankle and right wrist. He had a long standing history of seropositive rheumatoid arthritis (RA) with rheumatoid factor (RF) [RF positive: 101 (normal value $<15$ IU/ $\mathrm{mL})$ ], anti-cyclic-citrullinated peptide (CCP) [Anti-CCP: 340 (normal value $<20 \mathrm{U} / \mathrm{mL}$ )] which was not under control with non-biologic Disease Modifying Anti Rheumatic Drugs (DMARDS) such as hydroxyl-chloroquine, sulfasalazine or 
methotrexate. Owing to his inadequate response to the present medications, he was planned to put on biologic DMARD, rituximab. As a part of mandatory protocol to detect latent tuberculosis in patients with rituximab, our patient underwent high resolution Computed Tomography (HRCT) which showed fibrotic bronchiectatic changes. His ultrasound abdomen showed grade - II parenchymal changes in kidney; baseline liver and renal functions were normal. Rifampin $450 \mathrm{mg}$ and isoniazid 300 $\mathrm{mg}$ in combination with pyridoxine was started for six months as TB prophylaxis. Two days later one gram injection rituximab preceded by injection methyl prednisolone was administered. Patient was subsequently discharged on deflazacort, hydroxy-chloroquine, sulfasalazine, methotrexate, febuxostat, cilnidipine, pantoprozole, rifampin and isoniazid.

Two days later, patient was admitted with acute onset of fever and shortness of breath. On investigations, he was detected to have oliguria, jaundice, renal failure, elevated SGOT (554 mg/ dL) and SGPT (333 mg/dL). Provisional diagnosis was rifampin induced hepatitis and Acute Kidney Injury (AKI). Suspecting it to be sepsis (high procalcitonin: $150 \mu \mathrm{g} / \mathrm{L}$ ) he was started on injectable meropenam (1g twice daily) and levofloxacin (750 mg every third day). Shortness of breath was managed with inhaled steroids and $\beta 2$ agonists nebulization and oral acebrophylline (containing ambroxol and theophylline). Patient was put on liver and renal protectants (ursodeoxycholic acid and nephrocaps). All the non-biologic DMARDS were put on hold and patient was solely on injectable methyl prednisolone for control of RA. The renal function was found to be deteriorating (rising serum creatinine and nil urinary output) [Table 1], for which hemodialysis was performed.

After stabilization, he underwent renal biopsy which showed acute tubular interstitial nephritis. His renal condition got improved after three weeks of alternate day dialysis and he started passing urine.

\section{Discussion}

Acute interstitial nephritis is an important cause of acute renal failure resulting from immune-mediated tubule-interstitial injury, commonest cause being drugs. It is important to distinguish drug-induced interstitial nephritis from acute tubular necrosis, because early steroid administration can improve renal dysfunction caused by acute interstitial

Table 1: Significant changes in laboratory parameters.

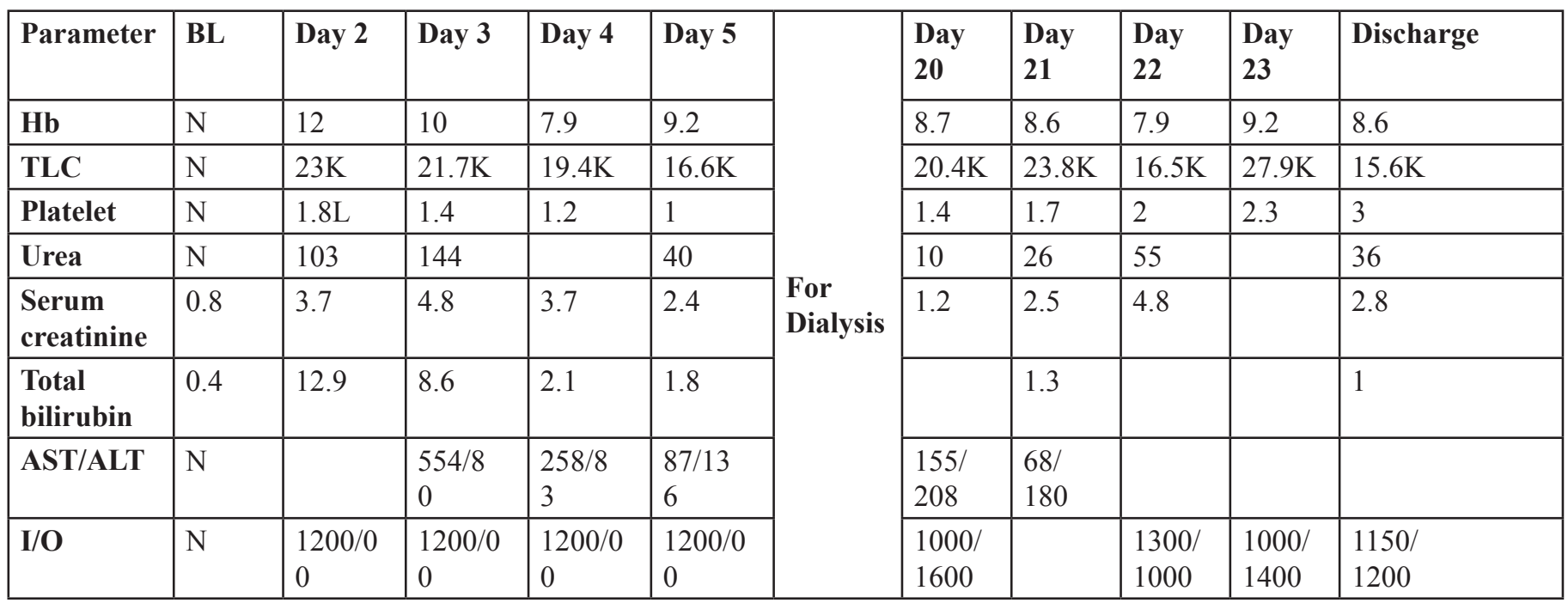

BL: baseline; Hb: hemoglobin; AST: aspartate transaminase; ALT: alanine transaminase; I/O: intake/output. 
nephritis. A reversible cause of renal failure due to acute interstitial renal inflammation is failure to recognize drug induced nephritis which can result in renal failure in $50 \%$ of the cases [6]. The rifampin component of the standard anti-TB regimen of isoniazid, rifampin, and pyrazinamide is most likely responsible for the onset of symptoms seen in this patient.

The mechanism of rifampin-induced AKI is not well established. Several studies suggest that it is either a type II or type III hypersensitivity reaction induced by rifampin antigens in which anti-rifampin antibodies form immune complexes that are deposited in renal vessels, the glomerular endothelium, and the interstitial area [7]. These reactions cause two different pathologic changes in the kidneys. The deposition of immune complexes in the vessels causes vascular constriction and tubular ischemia, leading to acute tubular necrosis, whereas the deposition of immune complexes in the interstitial area leads to acute interstitial nephritis. Renal biopsy remains the gold standard for diagnosis, but it may not be required in mild cases or when clinical improvement is rapid after removal of an offending agent or medication. The renal biopsies performed in several studies with a total of 106 patients reveal that the most common pathologies are acute interstitial nephritis (54\%) and acute tubular necrosis (38\%) [8]. The patient in this case was also treated with rituximab for his rheumatoid arthritis.

It is difficult to diagnose interstitial nephritis based on clinical and laboratory findings, and renal biopsy is required for a definitive diagnosis which was suggestive of acute tubular interstitial nephritis in our case.

\section{Conclusion}

Acute tubulo-interstitial nephritis should always be included in the differential diagnosis in a patient with acute kidney injury and prominent renal tubular damage following the initiation of rifampin. Drug discontinuation, renal biopsy followed by steroids at an early stage could produce a favourable renal outcome.

Contributors: MR, VP: Manuscript writing and literature review; MD: Manuscript revision and literature review. All authors were involved in case management. MR will act as guarantor. All authors approved the final version of the manuscript.

Funding: None; Competing interests: None stated.

\section{References}

1. Prasad R, Mukerjee PK. Rifampicin-induced thrombocytopenia. Ind J Tuberc. 1989;36:44-45.

2. Thangamani M, Matcha J, Edwin MF, Muthusethupathi MA. Acute Renal Failure Due to Rifampicin. A study of 25 patients. Am J Kidney Dis. 2002;40:690-696.

3. Poole G, Stradling P, Worlledge S. Potentially serious side effects of high dose twice weekly rifampicin. BMJ. 1971;3:343-347.

4. Shantha T, Nazareth O, Krishnamoorthy MS, Balasubramanian R, Vijayan VK, et al. Treatment of Pulmonary Tuberculosis with short course chemotherapy in South India-5 year follow up. Tubercle. 1989;70:229234.

5. Jawahar MS, Sivasubramanian S, Vijayan VK, Ramakrishnan CV, Paramasivan CN, Selvakumar V, Paul S, et al. Short course Chemotherapy for tuberculous lymphadenitis in children. BMJ. 1990;301:359-362.

6. Nishijima T, Yazaki H, Hinoshita F, Tasato D, Hoshimoto $\mathrm{K}$, Teruya $\mathrm{K}$, et al. Drug-induced acute interstitial nephritis mimicking acute tubular necrosis after initiation of tenofovir-containing antiretroviral therapy in patient with HIV-1 infection. Intern Med. 2012;51(17):24692471.

7. Muthukumar T, Jayakumar M, Fernando EM, Muthusethupathi MA. Acute renal failure due to rifampicin: a study of 25 patients. Am J kidney Dis. 2002;14(4):690-696.

8. Chang $\mathrm{CH}$, Chen $\mathrm{YF}, \mathrm{Wu} \mathrm{VC}$, Shu CC, Lee CH, Wang JY, et al. Acute kidney injury due to anti-tuberculosis drugs: a five-year experience in an aging population. BMC Infect Dis. 2014;14:23. 